\title{
Silicon Photonics Circuit for Tunable Low-Phase Noise RF Generation up to W-band
}

\author{
Antonio Malacarne \\ Photonic Networks \& Technologies \\ National Laboratory \\ CNIT \\ Pisa, Italy \\ antonio.malacarne@cnit.it
}

\author{
Antonio D'Errico \\ Ericsson Research \\ Ericsson \\ Pisa, Italy \\ antonio.d.errico@ericsson.com
}

\author{
Fabio Falconi \\ TeCIP Institute \\ Sant'Anna School of Advanced Studies \\ Pisa, Italy \\ fabio.falconi@santannapisa.it
}

\author{
Antonella Bogoni \\ TeCIP Institute \\ Sant'Anna School of Advanced Studies \\ Pisa, Italy \\ antonella.bogoni@santannapisa.it
}

\author{
Alessandra Bigongiari \\ Ericsson Research \\ Ericsson \\ Pisa, Italy \\ alessandra.bigongiari@ericsson.com

Claudio Porzi
TeCIP Institute
Sant'AnnaSchool of Advanced Studies
Pisa, Italy
claudio.porzi@santannapisa.it \\ Claudio Porzi \\ claudio.porzi@santannapisa.it
}

\begin{abstract}
This paper reports on the performance of a circuit monolithically integrated in Silicon-on-insulator (SOI) platform, employed for photonics-based carrier radiofrequency multiplication. An applied reference clock at $18.5 \mathrm{GHz}$ is successfully multiplied up to $92.5 \mathrm{GHz}$ (fivefold) with low additional phase noise. The SOI circuit includes a high-speed electro-optic phase modulator employed for optical spectral comb (OSC) generation and a thermally tunable distributed feedback resonator filter for selecting one of the OSC mode. Beating of the input laser mode with the mode selected by the filter into a high-speed photodiode generates the target output carrier frequency. By suitably tuning the clock frequency and the integrated optical filter, the solution permits reconfigurable frequency generation up to $\mathrm{W}$-band and, potentially, beyond. Experimental results demonstrate that the generated multiple carrier frequencies exhibit phase noise performance comparable with the one of the clock source.
\end{abstract}

Keywords-Photonics RF generation, Silicon photonics, microwave photonics, photonic processing, integrated optical filter, phase noise, time jitter

\section{INTRODUCTION (HEADING 1)}

Reconfigurable radiofrequency (RF) transmitters able to operate in the whole $0.3-300 \mathrm{GHz} R F$ domain find application in many fields such as $5 \mathrm{G} / 6 \mathrm{G}$ communications, radar/imaging systems and electronic warfare [1]-[3]. Fifth generation (5G) wireless technology in mobile communication networks plans the utilization of the $24-100 \mathrm{GHz}$ mm-wave range, whereas automotive radar standards already reach the $77-81 \mathrm{GHz}$ one [4]. In addition, various sub-ranges of W-band (75-110 GHz) have been allocated as "lightly licensed" bands for multigigabit wireless communications and satellite communications [4]. For both wireless communication and radar applications, higher carrier frequency enables larger bandwidth (for radar this translates into better resolution, for telecom into larger data rate) and smaller antenna size. The higher atmospheric attenuation of higher frequency makes this choice robust to inter-cell interference and suitable for distances up to a few kilometers. All those features are fully compatible with the emerging centralized radio access network (C-RAN) architectures where, by sharing a centralized base station for signal processing (generation, up/down conversion, etc.), remoting of small-size simplified antenna sites is possible through optical fiber links [5]. However, in such systems, phase noise (PN) of the generated

This work was partly sponsored by Ericsson Research and supported by the POINTING project founded by the Italian Space Agency. mm-wave carriers has a severe impact, especially for multicarrier signals with small subcarrier spacing, as envisioned in $5 \mathrm{G}$ wireless systems [6],[7].

In such a context, photonic techniques based on heterodyne detection of two laser carriers in a high-speed photodiode (PD) have long been proposed for generating high-frequency RF waves [8],[9], with benefits in terms of broad bandwidth offered by photonics and possibility of lowloss transport of the mm-wave signal through an optical fiber distribution network. Excellent PN performance have been achieved by selecting the beating optical modes out from a mode-locked fiber laser [10]. Though, such solution prevents integration and exhibits a high complexity. Other solutions realize dual-wavelength laser sources [11]. However, in those cases the use of InP platform is not particularly suitable for large volume production, the solution suffers from large phase noise [12] and/or fixed lasers frequency detuning. An alternative way to achieve PN performance comparable with a high-quality lower-frequency reference clock, is to perform frequency multiplication. It can be done through electrical phase-locked loops (PLLs) [13],[14], but non-idealities of electrical/electronic circuits (parasitic effects, amplifier noise etc.) push the operating conditions far from theoretical predictions [15]. In the optical domain, two optical beating tones can be conveniently selected from an optical spectral comb (OSC) generated through electro-optic modulation of a laser source (LS), driven by a reference RF clock. In this case, the quality of the generated RF tone depends on the purity of the lower-frequency reference, whereas the optical PN and linewidth due to the LS gets canceled during the beating process at the PD [16]. Mode selection can be performed through several types of optical filter, e.g. arrayed-waveguide gratings (AWGs) [17], Fabry-Perot filters (FPFs) [18], liquid crystal-on-silicon (LCoS) matrixes [19] etc.. However, so far, a monolithically integrated chip including OSC generation, mode selection and recombination, has not been implemented.

In this work, a monolithic silicon photonic integrated circuit (PIC) is successfully employed for reconfigurable RF frequency multiplication up to the W-band $(75-110 \mathrm{GHz})$, starting from an $18.5-\mathrm{GHz}$ reference frequency. The PIC incorporates OSC generation through a high-speed electrooptic phase modulator (PM), spectral mode selection through a $4^{\text {th }}$-order distributed feedback reflector (DFBR) bandpass filter [20], and its subsequent recombination with the original laser mode at the PIC output. OSC generation is driven by the $18.5-\mathrm{GHz}$ reference signal, and fivefold 
frequency multiplication, achieving a frequency of $92.5 \mathrm{GHz}$, is successfully demonstrated with limited additional PN with respect to the reference clock. The proposed method is CMOS compatible, reconfigurable mode selection is achieved through thermal tuning of the DFBR filter and a complete PN and time jitter analysis is reported for each generated carrier RF. The tolerance of the scheme to the optical frequency stability of the laser is also attested.

\section{EXPERIMENTAL SETUP}

The experimental setup employed to test the PIC is reported in Fig. 1. The circuit has been implemented in silicon-on-insulator (SOI) platform through a standard multi

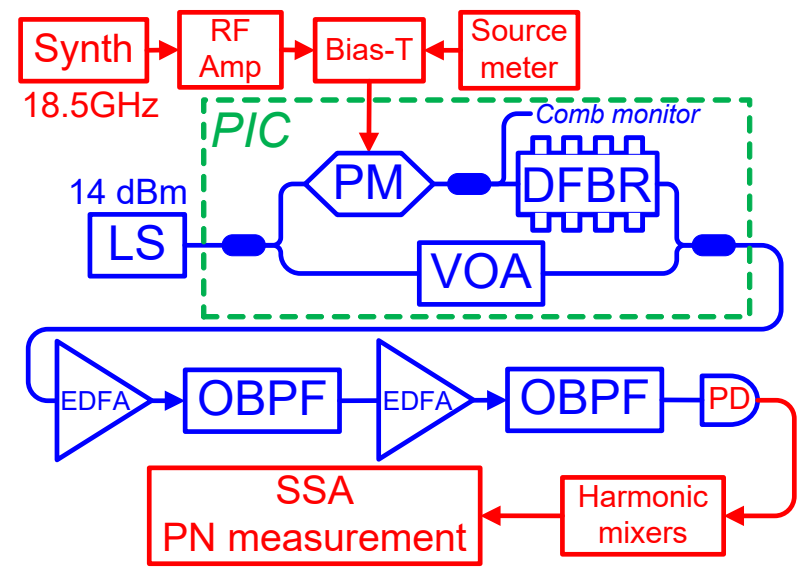

Fig. 1 Experimental setup

project wafer service [21]. The temperature of the whole PIC is kept constant by a Peltier cell controlled by a thermistorbased feedback. The mask layout of the employed structure and a picture of the PIC hosting the structure, are reported in Fig. 2. A C-band laser source (LS) with emission power of $14 \mathrm{dBm}$ is injected at the PIC input through an on-chip grating coupler (GC). Light is then split in two arms through a multimode interference (MMI) splitter. The upper arm includes the phase modulator (PM) employed for optical spectral comb (OSC) generation and, through a second MMI, the PM output can be monitored through a dedicated port, namely 'comb monitor'. The lower arm embeds a variable optical attenuator (VOA) consisting of a MZI structure with a thermal phase shifter on each arm.

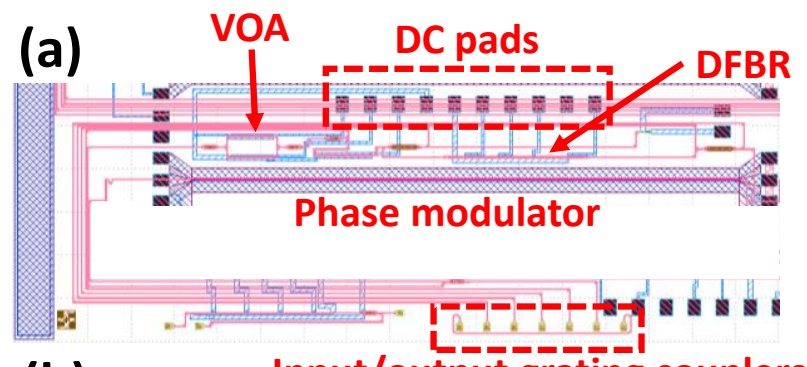

(b)

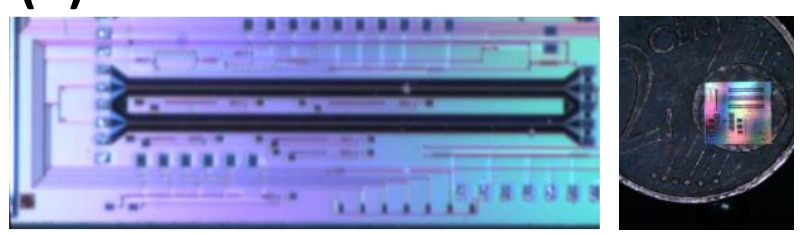

Fig. 2 (a) Mask layout of the employed scheme included in the fabricated PIC. (b) Picture of part of the fabricated PIC on the left and its dimension compared with a 2 cents coin on the right.
An RF synthesizer (Synth) generates a continuous wave with an $18.5 \mathrm{GHz}$-frequency, acting as the lower-frequency

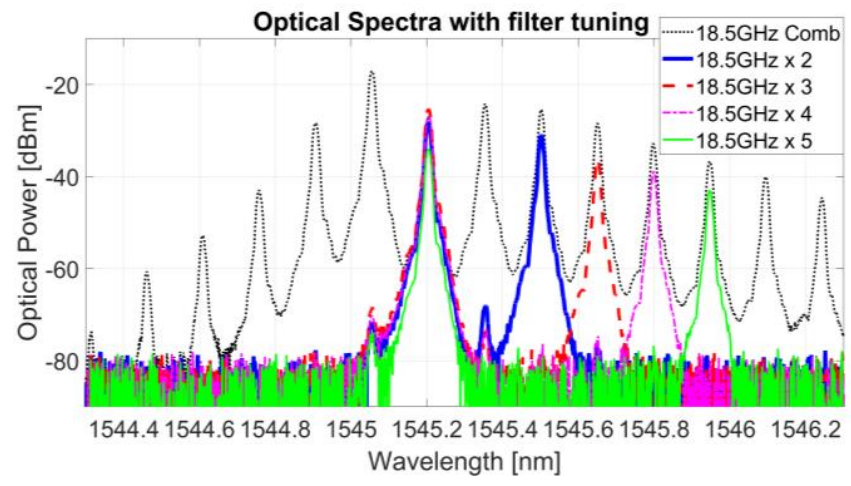

Fig. 3 OSC spectrum together with PIC output spectrum for each obtained frequency multiplication factor.

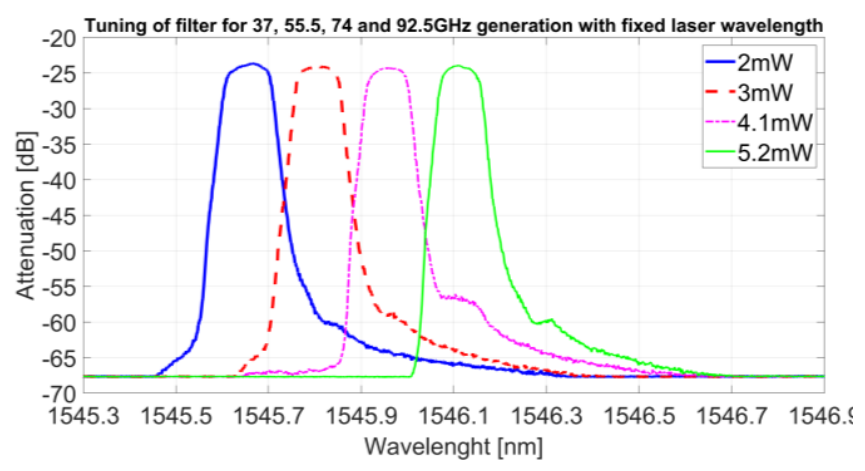

Fig. 4 DFBR filter response to achieve frequency multiplication from $\times 2$ up to $\times 5$ of the $18.5 \mathrm{GHz}$ clock frequency.

clock and employed for driving the PM with a power of $27 \mathrm{dBm}$ after RF amplification. The SOI PM is designed to exhibit a $20 \mathrm{GHz}$ electro-optical bandwidth. It is based on plasma dispersion effect in a doped silicon rib waveguide embedding a lateral pn-junction and operates in carrierdepletion regime. To set the modulator working condition, a proper DC reverse bias voltage $(3.2 \mathrm{~V}$ for maximizing the OSC generation efficiency) is coupled to the RF clock through a bias-tee. To achieve high-frequency RF carrier generation, one OSC mode is first selected by the following DFBR tunable filter and then coupled with the input laser mode through a MMI coupler. The two coupled optical modes are then extracted from the PIC through an output GC and delivered to a $100-\mathrm{GHz}$ photodiode (PD), for generating a beating RF carrier with a frequency equal to their spectral spacing, that is a multiple of the clock frequency, i.e. at $N \times 18.5 \mathrm{GHz}$, being $N$ a positive integer representing the frequency multiplication factor with respect to the clock. After photodetection, a Signal Source Analyzer (SSA) is employed (Agilent E5052A) for phase noise (PN) measurement. A Microwave Downconverter (E5053A) and additional external harmonic mixers are used to extend the bandwidth limitation of the instrument up to $110 \mathrm{GHz}$. To compensate for the large electrical loss due to the harmonic mixers and the high optical loss introduced by the PIC ( $4 \mathrm{~dB}$ of coupling loss at each input/output GC, $5 \mathrm{~dB}$ of PM insertion loss, $3 \mathrm{~dB}$ of loss for each traversed MMI), a double stage of optical amplification through erbium-doped fiber amplifiers (EDFAs) and tunable optical bandpass filters (OBPF) for removing out-of-band amplified spontaneous emission, is inserted before the PD. Fig. 3 shows the optical spectrum of the generated OSC (dotted thin black line) at the comb monitor port (Fig. 1). 
Through the DFBR filter, one of the comb mode is selected and coupled with the original laser mode at the GC output. The passband filter loss is $2 \mathrm{~dB}$, with a $1 \mathrm{~dB}$-band of $10 \mathrm{GHz}$ and adjacent mode rejection higher than $35 \mathrm{~dB}$. The DFBR passband can be tuned across a range of $6 \mathrm{~nm}$, through four thermal shifters, individually heating each of the four coupled cavities in the structure [20]. The VOA on the lower arm of the PIC is set in order to maximize the AC power at the PD output. In fact, due to the two EDFAs spectral gain and saturation regime, a too higher power of the laser mode with respect to the mode selected by the filter, induces a too high DC term at the PD output, eventually limiting the sensitivity of the PN measurement.

Fig. 4 shows the DFBR optical filter response when thermally tuning it so as to select an OSC mode $37,55.5,74$ and $92.5 \mathrm{GHz}$ spaced from the input laser mode. The legend reports the corresponding average dissipated power on each thermal shifter, for tuning the filter. Obviously, each filter response in Fig. 4 corresponds to an obtained spectrum of Fig. 3.

\section{EXPERIMENTAL RESULTS}

Through the setup shown in Fig. 1, the phase noise power spectral density (PSD) of each obtained carrier frequency, namely 37, 55.5, 74 and $92 \mathrm{GHz}$, respectively corresponding to frequency multiplication factor of 2, 3, 4 and 5 with respect to the reference frequency of $18.5 \mathrm{GHz}$, has been properly measured in the range $1 \mathrm{kHz}-40 \mathrm{MHz}$ of frequency offset from the carrier frequency under test. The range has been chosen according to the requirements of modern wireless communication applications and considering the $40 \mathrm{MHz}$

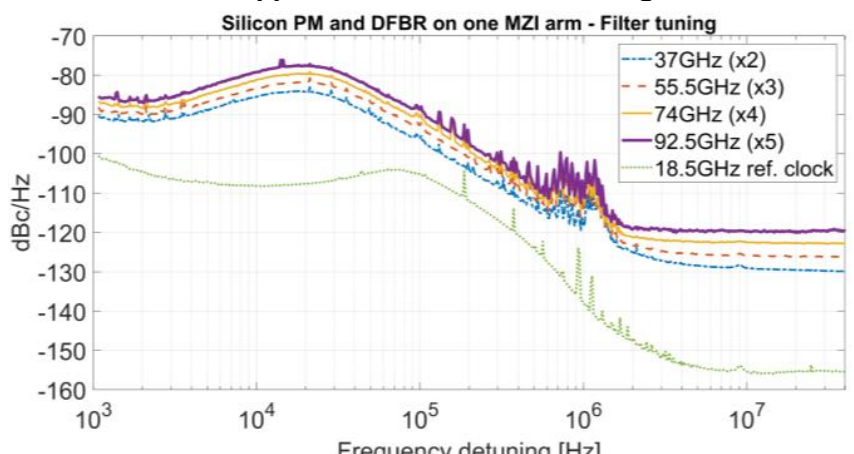

Fig. 5 Phase noise PSD of generated RF carrier at frequency of 37, 55.5, 74 and $92.5 \mathrm{GHz}$, together with the one of the employed clock signal at

$$
18.5 \mathrm{GHz} \text {. }
$$

instrument limit. Fig. 5 shows the PN PSD curves for multiplication factors up to 5, compared with the PN of the employed synthesizer when generating the $18.5 \mathrm{GHz}$ carrier. The increasingly higher noise level at larger multiplication factor is in accordance with the theoretical dependence of the $\mathrm{PN}$ level in $\mathrm{dBc} / \mathrm{Hz}$ on the square of $N$. For instance, this means that theoretically the PN curve of the $37 \mathrm{GHz}$ carrier should be $6 \mathrm{~dB}$ higher than the reference clock one. Analyzing the excess noise in $\mathrm{dBc} / \mathrm{Hz}$ among the reference curve and each multiplied-frequency component, only part of it is due to the higher analyzed frequency. The shape of each obtained PN curves is clearly different from the reference PN. In particular, a pronounced wide "bell-shaped" peak centered at about $20 \mathrm{kHz}$ is clearly visible and ascribed to additional PN to the optical mode selected by the DFBR filter. In fact, a Gaussian noise with the frequency content of such a bell-shaped additional PN term comes from each sourcemeter (2602B by Keithley) employed as current source for controlling each of the four thermal phase shifters responsible for DFBR filter tuning. Such a noise was measured to be $30 \mathrm{mV}_{\mathrm{pp}}$ through a real-time oscilloscope. Therefore, local temperature fluctuation at the heaters translates into a fluctuation of the DFBR filter spectral response. Such fluctuation induces phase fluctuation on the selected mode, therefore leading to an additional PN term on the generated RF tone after beating between filtered and laser modes, at the PD. According to this assumption, by tuning the filter towards higher-order OSC modes by increasing the power dissipated on the thermal shifters, from $2 \mathrm{~mW}$ for $37 \mathrm{GHz}$ generation up to $5.2 \mathrm{~mW}$ for $92.5 \mathrm{GHz}$ generation, the sourcemeter noise contribution increases because of the quadratic relation among current (or voltage) and corresponding power provided by the instrument.

As a final remark, the relatively high floor at detuning values $>10 \mathrm{MHz}$, represents a sensitivity limit of the measure, due to both the available signal power and its signal-to-noise ratio. The main limitation in that sense is given by the optical

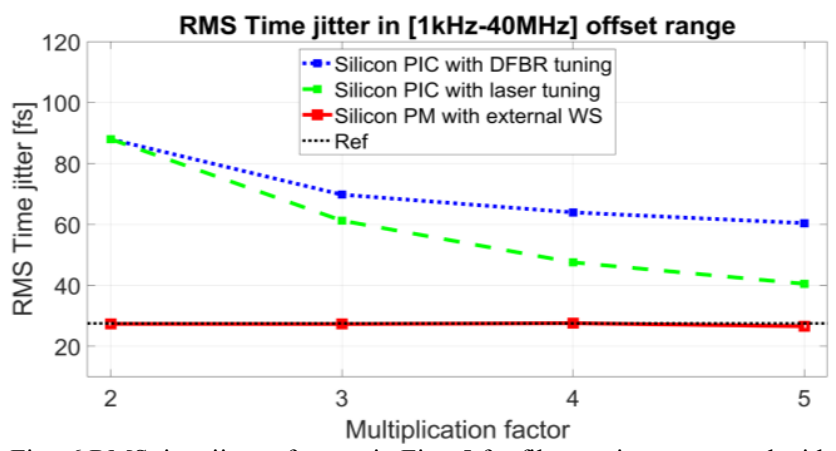

Fig. 6 RMS time jitter of traces in Fig. 5 for filter tuning, compared with the case where the input lasers tunes. In addition, the case where an external filter selects two comb modes in case of silicon PM.

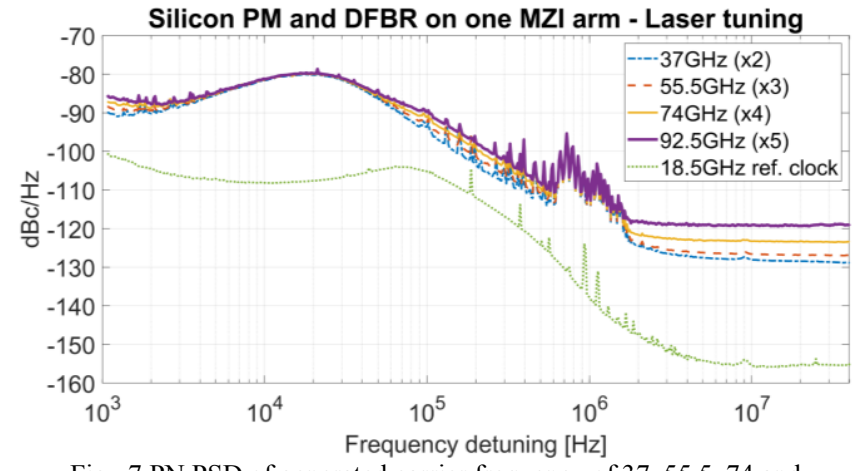

Fig. 7 PN PSD of generated carrier frequency of 37, 55.5, 74 and $92.5 \mathrm{GHz}$, together with the one of the clock signal at $18.5 \mathrm{GHz}$, in case of laser tuning, with $2 \mathrm{~mW}$ power dissipated on DFBR thermal shifters

insertion loss of the PIC, and the consequent optical SNR decrease caused by the use of the two EDFAs.

As a further performance evaluation, root-mean-square (RMS) time jitter (TJ) for each generated RF tone was measured in the same frequency offset range. Fig. 6 compares the obtained values when tuning the DFBR filter (dotted blue square) with the case where an external filter, i.e. a Finisar WaveShaper (WS) based on LCoS technology, selects the two optical modes at the comb monitor output (solid red square) (see Fig. 1). As for ideal frequency multiplication, in this last case $\mathrm{TJ}$ is independent of the multiplication factor, and equal to the TJ of the employed $18.5 \mathrm{GHz}$ reference clock (Ref). These results clearly indicate that the use of the DFBR filter leads to a worsening of the TJ. As the multiplication factor increases almost identically to the rate of the dissipated power on the thermal shifters, the decreasing trend of TJ with 
multiplication factor may be ascribed to some additional, and constant, disturb. Furthermore, the case of filter tuning has been compared with the case where the laser emission frequency has been tuned while keeping fixed the filter spectral response, with dissipated power of $2 \mathrm{~mW}$ (dashed green square). PN curves are shown in Fig. 7. In this case the TJ improvement with higher frequency multiplication is due to the constant phase noise induced by the filter. Such a trend is expected as, for increasing carrier frequency, the weight of a constant disturb on the TJ calculation decreases. Such an analysis reveals that, given a certain amount of noise provided by the current source driving the filter tuning process, better performance can be achieved by making use of a tunable laser instead of tuning the filter. In fact, this way, the dissipated power on thermal shifters employed for filter tuning can be kept as low as possible, mitigating the additional PN due to the noise of the current source driving the shifters. However, a fast tunable laser may not represent a cost-effective solution and, on the other hand, DFBR tuning speed was demonstrated to be as fast as about $200 \mu$ s (limited by the cooling process). Fast frequency agility represents an interesting feature for both reducing network latency for telecom, enabling tracking of high-speed moving targets in radar systems and high-speed RF scanning in case of electronic warfare [22].

As a last performance evaluation, TJ monitoring was performed when detuning the input laser emission frequency, in order to attest the system robustness to laser frequency instability. The result is reported in Fig. 8 where the measured $\mathrm{TJ}$ is reported in correspondence of the frequency detuning of the filtered mode with respect to the filter spectral response. Within a $7 \mathrm{GHz}$-wide detuning range a TJ worsening $<10 \%$ was observed, with such a value that increases up to $30 \%$ for a $12 \mathrm{GHz}$-wide detuning range.

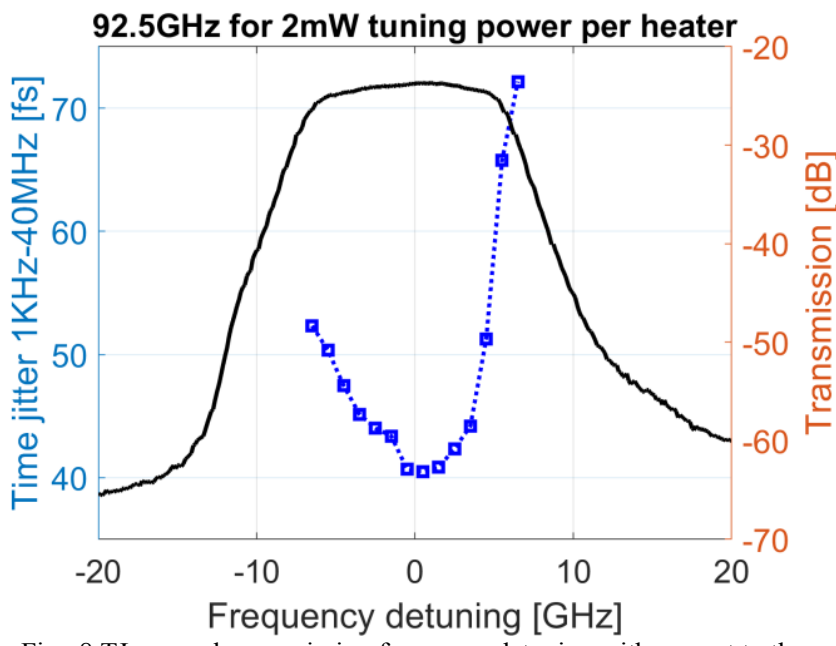

Fig. $8 \mathrm{TJ}$ versus laser emission frequency detuning with respect to the DFBR filter spectral response.

\section{CONCLUSION}

Photonics-based frequency multiplication of an $18.5 \mathrm{GHz}$ clock up to $92.5 \mathrm{GHz}$ (fivefold) is demonstrated using a monolithic silicon chip embedding an electro-optic phase modulator and a custom thermally tunable distributed feedback reflector filter. Limited additional phase noise is observed, due to the noise level of the current sources employed for driving the thermal shifters to achieve optical filter tuning. Improved performance is achieved by tuning the input laser frequency while fixing the filter spectral response with limited power dissipated on the thermal shifters.
However, in both cases, the introduced additional phase noise, and consequent time jitter value, can be limited by employing current sources with better noise performance. Robustness to laser emission frequency fluctuation within $7 \mathrm{GHz}$ is also attested. The solution is suitable for low-loss transport of lowphase noise W-band carriers through an optical fiber distribution network.

\section{REFERENCES}

[1] White Paper, "Cisco Visual Networking Index: Global Mobile Data Traffic Forecast Update 2017-2022” (2019).

[2] Moe Z. Win, et al., "History and Applications of UWB", Proceedings of the IEEE, Vol. 97, No. 2, February 2009

[3] Michael Frater and Michael J. Ryan "Electronic Warfare for the Digitized Battlefield", Artech House, January 2001

[4] Final draft ETSI EN $302 \quad 264 \quad$ V2.1.1 $\quad$ (2017-02) https://www.etsi.org/deliver/etsi_en/302200_302299/302264/02.01.0 1_30/en_302264v020101v.pdf

[5] China Mobile Research Institute, "C-RAN: The road towards green RAN” White Paper, ver. 2.3, Sep. 2013.

[6] C. Browning et al., "Gain-switched optical frequency combs for future mobile radio-over-fiber millimeter-wave systems," J. Lightw. Technol., vol. 36, no. 19, pp. 4602-4610, Oct. 2018.

[7] Ana Garcia Armada, "Understanding the effects of phase noise in orthogonal frequency division multiplexing (OFDM)," IEEE Trans. Broadcasting, vol. 47, no. 2, pp. 153 -159, June 2001

[8] A. J. Seeds, "Microwave photonics" IEEE Trans. Microw. Theory Tech., vol. 50, no. 3, pp. 877-887, 2002.

[9] T. Nagatsuma, "Generating millimeter and terahertz waves" IEEE Microw. Mag., vol. 10, no. 4, pp. 64-74, 2009.

[10] G. Serafino et al., "Phase and amplitude stability of ehf-band radar carriers generated from an active mode-locked laser" J. Lightw. Technol., vol. 29, no. 23, pp. 3551-3559, 2011.

[11] G. Carpintero et al., "Microwave photonic integrated circuits for millimeter-wave wireless communications," J. Lightw. Technol., vol. 32, no. 20, pp. 3495-3501, 2014

[12] J. Hulme et al., "Fully integrated microwave frequency synthesizer on heterogeneous silicon-iii/v," Opt. Express, vol. 25, no. 3, pp. 24222431, Feb 2017

[13] W. P. Robins, Phase noise in signal sources: theory and applications. vol.9. IET, 1984

[14] Collins, Ian. "Phase-Locked Loop (PLL) Fundamentals", Analog Dialogue 52-07, July 2018

[15] A. Elkholy et al., "A 6.75-8.25-GHz -250-dB FoM Rapid ON/OFF Fractional-N Injection-Locked Clock Multiplier" IEEE Journal of Solid-State Circuits, vol. 53, no. 6, pp. 1818-1829, 2018.

[16] L. Goldberg ; R.D. Esman ; K.J Williams, "Generation and control of microwave signals by optical techniques", IEE Proceedings $J$ (Optoelectronics), Vol. 139, no. 4, p. 288-295, 1992

[17] H. Song et al., "Broadband-frequency-tunable sub-terahertz wave generation using an optical comb, awgs, optical switches, and a unitraveling carrier photodiode for spectroscopic applications," J. Lightw. Technol., vol. 26, no. 15, pp. 2521-2530, 2008.

[18] R. Criado et al., "Continuous-wave sub-thz photonic generation with ultra-narrow linewidth, ultra-high resolution, full frequency range coverage and high long-term frequency stability," IEEE Trans Terahertz Sci Technol., vol. 3, no. 4, pp. 461-471, 2013.

[19] Koenig et al., "Wireless sub-thz communication system with high data rate," Nature photonics, vol. 7, no. 12, pp. 977-981, 2013.

[20] C. Porzi et al., "Silicon photonics high-order distributed feedback resonators filters," IEEE J. Quantum Electron., vol. 56, no. 1, pp. 1-9, 2020 .

[21] https://www.imec-int.com/en/integrated-photonics

[22] Ghelfi, P, Laghezza F., Bogoni A., "Photonics for Radar Networks and Electronic Warfare Systems" Editors: IET SciTech Book Series: Radar, Antennas and Electromagnetics, 2019, ISBN: 9781785613760

[23] F. Falconi1 et al., "Photonics-based tunable 1-50 GHz RF transmitter on silicon chip", Proc. Optical Fiber Communication Conference, Tu5F.2, 2021 